(c) 2010 IEEE. Personal use of this material is permitted. Permission from IEEE must be obtained for all other uses, in any current or future media, including reprinting/republishing this material for advertising or promotional purposes, creating new collective works, for resale or redistribution to servers or lists, or reuse of any copyrighted component of this work in other works. 


\section{Joint Source and Relay Optimization for Parallel MIMO Relays Using MMSE-DFE Receiver}

\author{
Apriana Toding \\ Dept. Electrical Computer Engr. \\ Curtin University of Technology \\ Bentley, WA 6102, Australia \\ Muhammad R. A. Khandaker \\ Dept. Electrical Computer Engr. \\ Curtin University of Technology \\ Bentley, WA 6102, Australia
}

\author{
Yue Rong \\ Dept. Electrical Computer Engr. \\ Curtin University of Technology \\ Bentley, WA 6102, Australia \\ Email: y.rong@curtin.edu.au
}

\begin{abstract}
In this paper, we study the optimal structure of the source precoding matrix and the relay amplifying matrices for multiple-input multiple-output (MIMO) relay communication systems with parallel relay nodes. In particular, a nonlinear decision feedback equalizer (DFE) is used at the destination node, and the minimal mean-squared error (MMSE) criterion is used to detect the transmitted signal at each stream. We show that the optimal source precoding matrix and the optimal relay amplifying matrices have a beamforming structure. Using such optimal source and relay matrices and the MMSE-DFE receiver, a joint source and relay power loading algorithm is developed to minimize the MSE of the signal waveform estimation. Compared with existing algorithms for parallel MIMO relay networks, the proposed source and relay design together with the MMSE-DFE receiver has a significant improvement in the system bit-errorrate performance.
\end{abstract}

Index Terms-multiple-input multiple-output (MIMO), relay networks, parallel relay, minimum mean-squared error (MMSE), decision feedback equalizer (DFE).

\section{INTRODUCTION}

It is well known that relay techniques are very efficient in enhancing the coverage and the energy efficiency of wireless communication systems [1], [2]. When nodes in the relay system are installed with multiple antennas, we call such system multiple-input multiple-output (MIMO) relay communication system. Recently, MIMO relay communication systems have attracted much research interest and provided significant improvement in terms of both spectral efficiency and link reliability [3]-[14]. Many works have studied the optimal relay amplifying matrix for the source-relay-destination channel. In [5] and [6], the optimal relay amplifying matrix maximizing the mutual information (MI) between the source and destination was derived assuming that the source covariance matrix is an identity matrix. In [7] and [8], the relay amplifying matrix was designed to minimize the mean-squared error (MSE) of the signal waveform estimation at the destination.

A few research has studied the jointly optimal structure of the source precoding matrix and the relay amplifying matrix for the source-relay-destination channel. In [9], both the source covariance matrix and the relay amplifying matrix were jointly designed to maximize the source-destination MI. In [10] and [11], a unified framework was developed to jointly optimize the source precoding matrix and the relay amplifying matrix for a broad class of objective functions. All the works [5]-[11] focus on MIMO relay systems with a single relay node at each hop.

MIMO relay systems with multiple parallel relay nodes have been investigated in [12] and [13]. In [12], the optimal relay amplifying matrices are developed to minimize the MSE of the signal waveform estimation. However, the source precoding matrix is not optimized in [12]. In [13], the authors investigated the jointly optimal structure of the source precoding matrix and the relay amplifying matrices when a linear minimal MSE (MMSE) receiver is used at the destination.

In this paper, we study MIMO parallel relay systems with a nonlinear decision feedback equalizer (DFE) at the destination. In particular, the MMSE criterion is used to estimate the transmitted signal at each stream. We call such receiver an MMSE-DFE receiver. We develop the jointly optimal source precoding matrix and relay amplifying matrices for MIMO parallel relay systems with nonlinear MMSE-DFE receiver. Simulation results show that the proposed source and relay matrices together with the MMSE-DFE receiver yield a significant bit-error-rate (BER) improvement compared with the linear MMSE based relay algorithm developed in [13].

We would like to mention that for MIMO relay system with a single relay node, the optimal source and relay matrices using the nonlinear MMSE-DFE receiver have been developed in [14]. This paper generalizes the relay design from a single relay node case to multiple parallel relay nodes scenario.

The rest of this paper is organized as follows. The system model is described in Section II. In Section III we study the optimal structure of the source and relay matrices. Section IV shows the simulation results. Conclusions are drawn in Section V.

\section{System MODEL}

Fig. 1 illustrates a two-hop MIMO relay communication system consisting of one source node, $K$ parallel relay nodes, and one destination node. We assume that the source and destination nodes have $N_{s}$ and $N_{d}$ antennas, respectively, and each relay node has $N_{r}$ antennas. The generalization to the system with different number of antennas at each relay node is straightforward. To efficiently exploit the system hardware, each relay node uses the same antennas to transmit and receive 


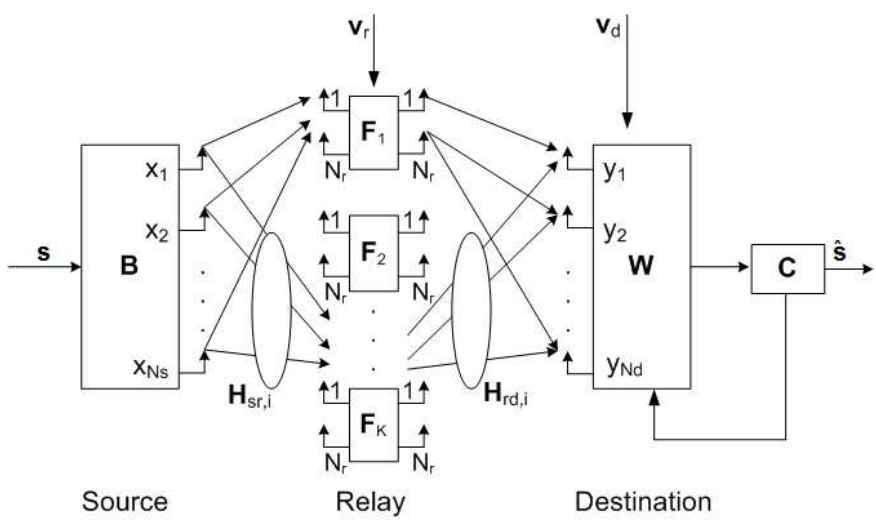

Fig. 1. Block diagram of a parallel MIMO relay communication system.

signals. Due to its merit of simplicity, we consider the amplifyand-forward relaying scheme at each relay.

The communication process between the source and destination nodes is completed in two time slots. In the first time slot, the $N_{b} \times 1$ modulated symbol vector $\mathbf{s}$ is linearly precoded as

$$
\mathbf{x}=\mathbf{B s}
$$

where $\mathbf{B}$ is an $N_{s} \times N_{b}$ source precoding matrix. We assume that $\mathrm{E}\left[\mathbf{s s}^{H}\right]=\mathbf{I}_{N_{b}}$, where $(\cdot)^{H}$ denotes matrix (vector) Hermitian transpose, E[.] stands for statistical expectation, and $\mathbf{I}_{n}$ is an $n \times n$ identity matrix. The precoded vector $\mathbf{x}$ is transmitted to the relay nodes and the received signal at the $i$ th relay node can be written as

$$
\mathbf{y}_{r, i}=\mathbf{H}_{s r, i} \mathbf{x}+\mathbf{v}_{r, i}, \quad i=1, \cdots, K
$$

where $\mathbf{H}_{s r, i}$ is the $N_{r} \times N_{s}$ MIMO channel matrix between the source and the $i$ th relay node, $\mathbf{y}_{r, i}$ and $\mathbf{v}_{r, i}$ are the received signal and the additive Gaussian noise vectors at the $i$ th relay node, respectively.

In the second time slot, the source node is silent, while each relay node transmits the amplified signal vector to the destination node as

$$
\mathbf{x}_{r, i}=\mathbf{F}_{i} \mathbf{y}_{r, i}, \quad i=1, \cdots, K
$$

where $\mathbf{F}_{i}$ is the $N_{r} \times N_{r}$ amplifying matrix at the $i$ th relay node. Thus the received signal vector at the destination node can be written as

$$
\mathbf{y}_{d}=\sum_{i=1}^{K} \mathbf{H}_{r d, i} \mathbf{x}_{r, i}+\mathbf{v}_{d}
$$

where $\mathbf{H}_{r d, i}$ is the $N_{d} \times N_{r}$ MIMO channel matrix between the $i$ th relay and the destination node, $\mathbf{y}_{d}$ and $\mathbf{v}_{d}$ are the total received signal and the additive Gaussian noise vectors at the destination node, respectively.

Substituting (1)-(3) into (4), we obtain

$$
\begin{aligned}
\mathbf{y}_{d} & =\sum_{i=1}^{K}\left(\mathbf{H}_{r d, i} \mathbf{F}_{i} \mathbf{H}_{s r, i} \mathbf{B} \mathbf{s}+\mathbf{H}_{r d, i} \mathbf{F}_{i} \mathbf{v}_{r, i}\right)+\mathbf{v}_{d} \\
& =\mathbf{H}_{r d} \mathbf{F} \mathbf{H}_{s r} \mathbf{B s}+\mathbf{H}_{r d} \mathbf{F} \mathbf{v}_{r}+\mathbf{v}_{d}
\end{aligned}
$$

where we define

$$
\begin{aligned}
\mathbf{H}_{s r} & \triangleq\left[\mathbf{H}_{s r, 1}^{T}, \mathbf{H}_{s r, 2}^{T}, \cdots, \mathbf{H}_{s r, K}^{T}\right]^{T} \\
\mathbf{H}_{r d} & \triangleq\left[\mathbf{H}_{r d, 1}, \mathbf{H}_{r d, 2}, \cdots, \mathbf{H}_{r d, K}\right] \\
\mathbf{F} & \triangleq \operatorname{bd}\left[\mathbf{F}_{1}, \mathbf{F}_{2}, \cdots, \mathbf{F}_{K}\right] \\
\mathbf{v}_{r} & \triangleq\left[\mathbf{v}_{r, 1}^{T}, \mathbf{v}_{r, 2}^{T}, \cdots, \mathbf{v}_{r, K}^{T}\right]^{T} .
\end{aligned}
$$

Here $(\cdot)^{T}$ denotes the matrix (vector) transpose, bd(.) stands for a block-diagonal matrix, $\mathbf{H}_{s r}$ is a $K N_{r} \times N_{s}$ channel matrix between the source node and all relay nodes, $\mathbf{H}_{r d}$ is an $N_{d} \times K N_{r}$ channel matrix between all relay nodes and the destination node, $\mathbf{v}_{r}$ is obtained by stacking the noise vectors at all the relays and $\mathbf{F}$ is the equivalent $K N_{r} \times K N_{r}$ block diagonal relay matrix. The diagram of the equivalent MIMO relay system described by (5) is shown in Fig. 2.

By introducing

$$
\overline{\mathbf{F}} \triangleq \mathbf{H}_{r d} \mathbf{F}
$$

the received signal vector at the destination can be equivalently written as

$$
\mathbf{y}_{d}=\overline{\mathbf{F}} \mathbf{H}_{s r} \mathbf{B} \mathbf{s}+\overline{\mathbf{F}} \mathbf{v}_{r}+\mathbf{v}_{d}=\overline{\mathbf{H}} \mathbf{s}+\overline{\mathbf{v}}
$$

where we define $\overline{\mathbf{H}} \triangleq \overline{\mathbf{F}} \mathbf{H}_{s r} \mathbf{B}$ as the effective MIMO channel matrix of the source-relay-destination link, and $\overline{\mathbf{v}}$ as the equivalent noise with $\overline{\mathbf{v}} \triangleq \overline{\mathbf{F}} \mathbf{v}_{r}+\mathbf{v}_{d}$. Compared with single-hop MIMO systems, in a parallel MIMO relay system, the channel matrix $\overline{\mathbf{H}}$ is a function of both $\mathbf{B}$ and $\mathbf{F}$, and the noise $\overline{\mathbf{v}}$ also depends on $\mathbf{F}$. This greatly complicates the system optimization issue as will be seen later.

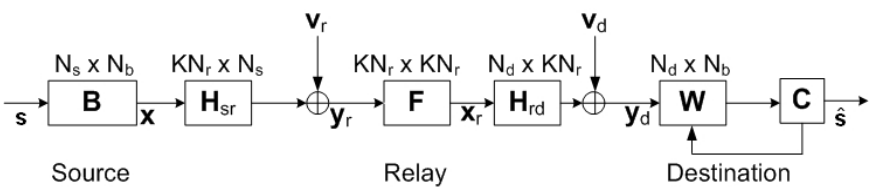

Fig. 2. Block diagram of the equivalent MIMO relay system.

\section{Optimal Source AND Relay Design}

At the destination node, a nonlinear DFE receiver is used to detect the source symbols successively with the $N_{b}$ th symbol detected first and the first symbol detected last. Assuming that there is no error propagation in the DFE receiver, the $k$ th source symbol is estimated as

$$
\hat{s}_{k}=\mathbf{w}_{k}^{H} \mathbf{y}_{d}-\sum_{l=k+1}^{N_{b}} c_{k, l} s_{l}, \quad k=1, \cdots, N_{b}
$$

where $\mathbf{w}_{k}$ is the feed-forward vector for the $k$ th symbol, and $c_{k, l}, l=k+1, \cdots, N_{b}$, are the feedback coefficients for the $k$ th symbol. By introducing $\mathbf{W}=\left[\mathbf{w}_{1}, \mathbf{w}_{2}, \cdots, \mathbf{w}_{N_{b}}\right], \hat{\mathbf{s}}=$ $\left[\hat{s}_{1}, \hat{s}_{1}, \cdots, \hat{s}_{N_{b}}\right]^{T}$, and an $N_{b} \times N_{b}$ strictly upper-triangle matrix $\mathbf{C}$ with nonzero elements $c_{k, l}$, we can represent (7) in matrix form as

$$
\hat{\mathbf{s}}=\mathbf{W}^{H} \mathbf{y}_{d}-\mathbf{C s}=\left(\mathbf{W}^{H} \overline{\mathbf{H}}-\mathbf{C}\right) \mathbf{s}+\mathbf{W}^{H} \overline{\mathbf{v}}
$$


where $\mathbf{W}$ and $\mathbf{C}$ are the feed-forward matrix and the feedback matrix of the DFE receiver, respectively. To minimize error of the signal estimation in (8), we get

$$
\mathbf{C}=\mathcal{U}\left[\mathbf{W}^{H} \overline{\mathbf{H}}\right]
$$

where $\mathcal{U}\left[\mathbf{W}^{H} \overline{\mathbf{H}}\right]$ denotes the strictly upper-triangular part of $\mathbf{W}^{H} \overline{\mathbf{H}}$. Substituting (9) back into (7), we obtain

$$
\hat{s}_{k}=\mathbf{w}_{k}^{H}\left([\overline{\mathbf{H}}]_{1: k}[\mathbf{s}]_{1: k}+\overline{\mathbf{v}}\right), \quad k=1, \cdots, N_{b}
$$

where $[\mathbf{a}]_{1: k}$ denotes a vector containing the first $k$ elements of vector $\mathbf{a}$, and $[\mathbf{A}]_{1: k}$ stands for a matrix containing the first $k$ columns of $\mathbf{A}$.

When the MMSE criterion is used to estimate each symbol, from (10) the feed-forward matrix $\mathbf{W}$ is given as

$$
\mathbf{w}_{k}=\left([\overline{\mathbf{H}}]_{1: k}[\overline{\mathbf{H}}]_{1: k}^{H}+\mathbf{C}_{\bar{v}}\right)^{-1} \overline{\mathbf{h}}_{k}, \quad k=1, \cdots, N_{b}
$$

where $\mathbf{C}_{\bar{v}}$ is the equivalent noise covariance matrix given by $\mathbf{C}_{\bar{v}}=\mathrm{E}\left[\overline{\mathbf{v}} \overline{\mathbf{v}}^{H}\right]=\overline{\mathbf{F}} \overline{\mathbf{F}}^{H}+\mathbf{I}_{N_{d}},(\cdot)^{-1}$ denotes the matrix inversion, and $\overline{\mathbf{h}}_{k}$ is the $k$ th column of $\overline{\mathbf{H}}$. Let us introduce the following QR decomposition

$$
\mathbf{G} \triangleq\left[\begin{array}{c}
\mathbf{C}_{\bar{v}}^{-1 / 2} \overline{\mathbf{H}} \\
\mathbf{I}_{N_{b}}
\end{array}\right]=\mathbf{Q R}=\left[\begin{array}{l}
\overline{\mathbf{Q}} \\
\mathbf{Q}
\end{array}\right] \mathbf{R}
$$

where $\mathbf{R}$ is an $N_{b} \times N_{b}$ upper-triangular matrix with all positive diagonal elements, $\mathbf{Q}$ is an $\left(N_{b}+N_{d}\right) \times N_{b}$ semi-unitary matrix with $\mathbf{Q}^{H} \mathbf{Q}=\mathbf{I}_{N_{b}}, \overline{\mathbf{Q}}$ is a matrix containing the first $N_{d}$ rows of $\mathbf{Q}$, and $\mathbf{Q}$ contains the last $N_{b}$ rows of $\mathbf{Q}$.

Using the QR decomposition (11), it has been shown in [14] that the feed-forward weight matrix $\mathbf{W}$, the feedback matrix $\mathbf{C}$ and the MSE matrix $\mathbf{E}=\mathrm{E}\left[(\hat{\mathbf{s}}-\mathbf{s})(\hat{\mathbf{s}}-\mathbf{s})^{H}\right]$ can be written as

$$
\mathbf{W}=\mathbf{C}_{\bar{v}}^{-1 / 2} \overline{\mathbf{Q}} \mathbf{D}_{R}^{-1}, \quad \mathbf{C}=\mathbf{D}_{R}^{-1} \mathbf{R}-\mathbf{I}_{N_{b}}, \quad \mathbf{E}=\mathbf{D}_{R}^{-2}
$$

where $\mathbf{D}_{R}$ is a matrix taking the diagonal elements of $\mathbf{R}$ as the main diagonal and zero elsewhere. Using (11) and (12), the joint source and relay optimization problem which minimizes the MSE of the signal waveform estimation can be formulated as

$$
\begin{array}{ll}
\min _{\overline{\mathbf{F}}, \mathbf{B}} & \operatorname{tr}\left(\mathbf{D}_{R}^{-2}\right) \\
\text { s.t. } & {\left[\begin{array}{c}
\mathbf{C}_{\bar{v}}^{-1 / 2} \overline{\mathbf{H}} \\
\mathbf{I}_{N_{b}}
\end{array}\right]=\mathbf{Q R}} \\
& \operatorname{tr}\left(\mathbf{B B}^{H}\right) \leq P_{s} \\
& \operatorname{tr}\left(\overline{\mathbf{F}}\left[\mathbf{H}_{s r} \mathbf{B B}^{H} \mathbf{H}_{s r}^{H}+\mathbf{I}_{N_{r}}\right] \overline{\mathbf{F}}^{H}\right) \leq P_{r}
\end{array}
$$

where $\operatorname{tr}(\cdot)$ stands for matrix trace, (15) is the transmit power constraint at the source node, while (16) is the power constraint at the output of $\mathbf{H}_{r d}$ [12], [13]. Here $P_{r}>0$ and $P_{s}>0$ are the corresponding power budgets.

Let $\mathbf{H}_{s r}=\mathbf{U}_{s} \boldsymbol{\Lambda}_{s} \mathbf{V}_{s}^{H}$ denote the singular value decomposition (SVD) of $\mathbf{H}_{s r}$, where the dimensions of $\mathbf{U}_{s}, \boldsymbol{\Lambda}_{s}, \mathbf{V}_{s}$ are $K N_{r} \times K N_{r}, K N_{r} \times N_{s}, N_{s} \times N_{s}$, respectively. We assume that the main diagonal elements of $\boldsymbol{\Lambda}_{s}$ are arranged in a decreasing order. We also introduce $M=\min \left(R_{h}, N_{b}\right)$, where $R_{h} \triangleq \min \left(\operatorname{rank}\left(\mathbf{H}_{s r}\right), \operatorname{rank}\left(\mathbf{H}_{r d}\right)\right)$ and $\operatorname{rank}(\cdot)$ denotes the rank of a matrix. Using the nonlinear MMSE-DFE receiver at the destination node of a parallel MIMO relay network, the optimal source precoding matrix and the relay amplifying matrices as the solution to the problem (13)-(16) are given by

$$
\overline{\mathbf{F}}=\mathbf{V} \boldsymbol{\Lambda}_{f} \mathbf{U}_{s, 1}^{H}, \quad \mathbf{B}=\mathbf{V}_{s, 1} \boldsymbol{\Lambda}_{b} \mathbf{V}_{r}^{H}
$$

where $\boldsymbol{\Lambda}_{f}$ and $\boldsymbol{\Lambda}_{b}$ are $M \times M$ diagonal matrices, $\mathbf{V}$ is any $N_{d} \times M$ semi-unitary matrix with $\mathbf{V}^{H} \mathbf{V}=\mathbf{I}_{M}, \mathbf{U}_{s, 1}$ and $\mathbf{V}_{s, 1}$ contain the leftmost $M$ vectors of $\mathbf{U}_{s}$ and $\mathbf{V}_{s}$, respectively, and $\mathbf{V}_{r}$ is an $N_{b} \times M$ semi-unitary matrix $\left(\mathbf{V}_{r}^{H} \mathbf{V}_{r}=\mathbf{I}_{M}\right)$ such that the QR decomposition in (14) holds. The proof of (17) is similar to the proof of Theorem 2 in [14].

From (17), we find that both $\overline{\mathbf{F}}$ and $\mathbf{B}$ have a beamforming structure. In particular, they jointly diagonalize the sourcerelay-destination channel matrix $\overline{\mathbf{H}}$ up to rotational matrices $\mathbf{V}$ and $\mathbf{V}_{r}$. It can be shown similar to [14] that the constraint (14) can be equivalently written as

$$
\mathbf{d}\left[\mathbf{D}_{R}\right] \prec \boldsymbol{\sigma}_{G}
$$

where $\prec$ stands for multiplicative majorization [15], $\sigma_{G}$ is a column vector containing singular values of $\mathbf{G}$, and $\mathbf{d}\left[\mathbf{D}_{R}\right]$ is a column vector containing all diagonal elements of $\mathbf{D}_{R}$. Let us denote $\lambda_{f, i}, \lambda_{s, i}, \lambda_{b, i}, i=1, \cdots, M$, as the main diagonal elements of $\boldsymbol{\Lambda}_{f}, \boldsymbol{\Lambda}_{s}, \boldsymbol{\Lambda}_{b}$, respectively. Using (17) and (18), the optimization problem (13)-(16) can be equivalently rewritten as

$$
\begin{aligned}
\min _{\left\{\lambda_{f, i}\right\},\left\{\lambda_{b, i}\right\}} & \operatorname{tr}\left(\mathbf{D}_{R}^{-2}\right) \\
\text { s.t. } \quad & \mathbf{d}\left[\mathbf{D}_{R}^{2}\right] \prec_{\mathrm{w}}\left[\left\{1+\frac{\left(\lambda_{f, i} \lambda_{s, i} \lambda_{b, i}\right)^{2}}{\lambda_{f, i}^{2}+1}\right\}^{T}, \mathbf{1}_{N_{b}-M}\right]^{T} \\
& \sum_{i=1}^{M} \lambda_{b, i}^{2} \leq P_{s} \\
& \sum_{i=1}^{M} \lambda_{f, i}^{2}\left[\left(\lambda_{s, i} \lambda_{b, i}\right)^{2}+1\right] \leq P_{r} \\
& \lambda_{b, i} \geq 0, \quad \lambda_{f, i} \geq 0, \quad i=1, \cdots, M
\end{aligned}
$$

where $\prec_{\mathrm{w}}$ stands for weakly multiplicative submajorization [15], $\{a\}$ stands for an $M \times 1$ vector $\left[a_{1}, a_{2}, \cdots, a_{M}\right]^{T}$, $\mathbf{1}_{N_{b}-M}$ denotes a $1 \times\left(N_{b}-M\right)$ vector with all 1 elements.

The problem (19)-(23) is highly nonconvex and a closedform solution is intractable to obtain. In the following, we develop an iterative method to obtain a numerical solution of the optimal $\left\{\lambda_{f, i}\right\}$ and $\left\{\lambda_{b, i}\right\}$. Let us define

$$
\begin{aligned}
& a_{i} \triangleq \lambda_{s, i}^{2}, \quad x_{i} \triangleq \lambda_{b, i}^{2}, \\
& y_{i} \triangleq \lambda_{f, i}^{2}\left[\left(\lambda_{s, i} \lambda_{b, i}\right)^{2}+1\right], \quad i=1, \cdots, M .
\end{aligned}
$$

Then using the definition of the operator $\prec_{w}$ in [15], the 
optimization problem (19)-(23) can be equivalently written as

$$
\begin{aligned}
\min _{\left\{x_{i}\right\},\left\{y_{i}\right\}} & -\sum_{i=1}^{M} \log \left(1+\frac{\frac{a_{i} x_{i} y_{i}}{a_{i} x_{i}+1}}{1+\frac{y_{i}}{a_{i} x_{i}+1}}\right) \\
\text { s.t. } & \sum_{i=1}^{M} x_{i} \leq P_{s} \\
& \sum_{i=1}^{M} y_{i} \leq P_{r} \\
& x_{i} \geq 0, \quad y_{i} \geq 0, \quad i=1, \cdots, M .
\end{aligned}
$$

For a fixed $\left\{y_{i}\right\}$ satisfying (27) and (28), the problem of optimizing $\left\{x_{i}\right\}$ can be written as

$$
\begin{array}{ll}
\min _{\left\{x_{i}\right\}} & \sum_{i=1}^{M} \log \left(\frac{a_{i} x_{i}+y_{i}+1}{a_{i} x_{i} y_{i}+a_{i} x_{i}+y_{i}+1}\right) \\
\text { s.t. } & \sum_{i=1}^{M} x_{i} \leq P_{s} \\
& x_{i} \geq 0, \quad i=1, \cdots, M .
\end{array}
$$

The Lagrangian function associated with the problem (29)-(31) can be written as

$$
\mathcal{L}=\sum_{i=1}^{M} \log \left(\frac{a_{i} x_{i}+y_{i}+1}{a_{i} x_{i} y_{i}+a_{i} x_{i}+y_{i}+1}\right)+\mu_{1}\left(\sum_{i=1}^{M} x_{i}-P_{s}\right)
$$

where $\mu_{1} \geq 0$ is the Lagrangian multiplier. Taking the derivative of (32) with respect to $x_{i}$ equal to zero, we obtain

$$
x_{i}=\frac{1}{2 a_{i}}\left[\sqrt{y_{i}{ }^{2}+\frac{4 a_{i} y_{i}}{\mu_{1}}}-y_{i}-2\right]^{\dagger}, \quad i=1, \cdots, M
$$

where $[x]^{\dagger} \triangleq \max (x, 0)$, and $\mu_{1}$ is the solution to the following nonlinear equation

$$
\sum_{i=1}^{M} \frac{1}{a_{i}}\left[\sqrt{y_{i}^{2}+\frac{4 a_{i} y_{i}}{\mu_{1}}}-y_{i}-2\right]^{\dagger}=2 P_{s} .
$$

In a similar fashion, for a fixed $\left\{x_{i}\right\}$ satisfying (26) and (28), we can update $\left\{y_{i}\right\}$ as

$$
y_{i}=\frac{1}{2}\left[\sqrt{a_{i}{ }^{2} x_{i}{ }^{2}+\frac{4 a_{i} x_{i}}{\mu_{2}}}-a_{i} x_{i}-2\right]^{\dagger}, \quad i=1, \cdots, M
$$

where $\mu_{2} \geq 0$ is the solutions to the following nonlinear equation

$$
\sum_{i=1}^{M}\left[\sqrt{a_{i}{ }^{2} x_{i}{ }^{2}+\frac{4 a_{i} x_{i}}{\mu_{2}}}-a_{i} x_{i}-2\right]^{\dagger}=2 P_{r}
$$

The iterative algorithm can be initialized at any random feasible $\mathbf{x}$ or $\mathbf{y}$. Since the conditional updates of $\left\{x_{i}\right\}$ and $\left\{y_{i}\right\}$ may either decrease or maintain but cannot increase the objective function in (25). Monotonic convergence of $\left\{x_{i}\right\}$ and $\left\{y_{i}\right\}$ to a locally optimum solution follows directly from this observation. After the convergence of the alternating algorithm, $\lambda_{f, i}$ and $\lambda_{b, i}$ can be obtained from (24) as

$$
\lambda_{f, i}=\sqrt{\frac{y_{i}}{a_{i} x_{i}+1}}, \quad \lambda_{b, i}=\sqrt{x_{i}}, \quad i=1, \cdots, M
$$

and then the optimal structure of $\overline{\mathbf{F}}$ and $\mathbf{B}$ is given by (17). The rotation matrix $\mathbf{V}_{r}$ in (17) can be computed using the numerical method developed in [16]. From (6), we have $\mathbf{H}_{r d, i} \mathbf{F}_{i}=$ $\mathbf{V} \boldsymbol{\Lambda}_{f} \mathbf{U}_{s, i}^{H}$, where matrix $\mathbf{U}_{s, i}^{H}$ contains the $(i-1) N_{r}+1$ to $i N_{r}$ columns of $\mathbf{U}_{s}^{H}$. Finally $\mathbf{F}_{i}=\left(\mathbf{H}_{r d, i}\right)^{+} \mathbf{V} \boldsymbol{\Lambda}_{f} \mathbf{U}_{s, i}^{H}$, $i=1, \cdots, K$, where $(\cdot)^{+}$denotes matrix pseudo-inverse.

\section{Simulations}

In this section, we study the performance of the proposed optimal joint source and relay beamforming algorithm for parallel MIMO relay systems with MMSE-DFE receiver at the destination. All simulations are conducted in a flat Rayleigh fading environment using the BPSK constellation, and the noises are i.i.d. Gaussian with zero mean and unit variance. The channel matrices have zero-mean entries with variances $\sigma_{s}^{2} / N_{s}$ and $\sigma_{r}^{2} /\left(K N_{r}\right)$ for $\mathbf{H}_{s r}$ and $\mathbf{H}_{r d}$, respectively. We vary the signal-to-noise ratio (SNR) in the source-to-relay link $\mathrm{SNR}_{\mathrm{S}}$ while fixing the SNR in the relay-to-destination link $\mathrm{SNR}_{\mathrm{r}}$ to $20 \mathrm{~dB}$. We transmit 1000 randomly generated bits in each channel realization, and the BER results are averaged through 200 channel realizations. Here we set $N_{b}=N_{s}=$ $N_{r}=N_{d}=3$.

In the first example, we simulate $K=3$ and compare the BER performance of the proposed optimal joint source and relay algorithm with the naive amplify-and-forward (NAF) algorithm where both the source and relay matrices are scaled identity matrices, and the joint source and relay algorithm for parallel MIMO relay systems where a linear MMSE receiver is applied at the destination [13]. The latter algorithm is denoted as MMSE algorithm. From Fig. 3, it can be seen that the NAF algorithm has the worst performance. The proposed algorithm outperforms the other two approaches.

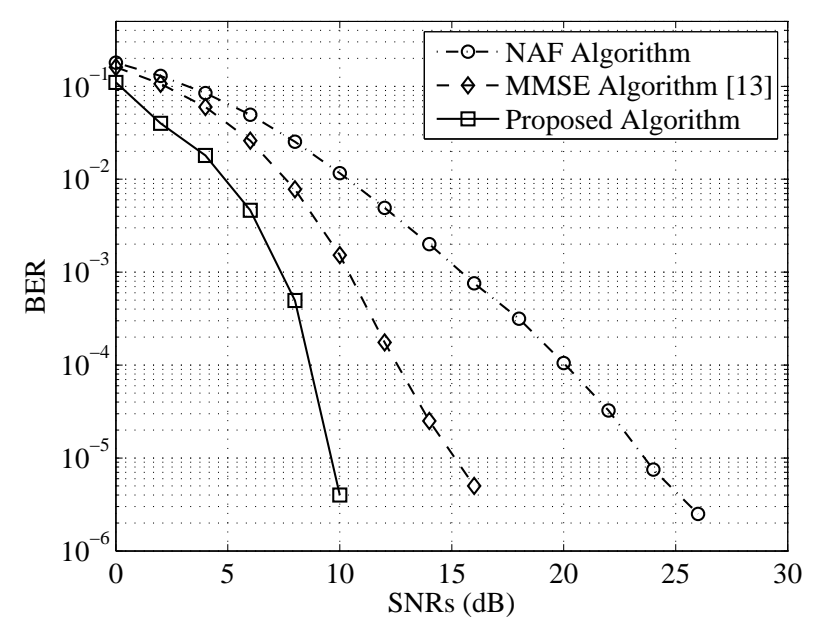

Fig. 3. Example 1. BER versus $\mathrm{SNR}_{\mathrm{s}}$ with $K=3$.

In the second example, we study the effect of the number of relays to the system BER performance using the proposed algorithm. Fig. 4 shows the BER performance with $K=2,3$, and 5. It can be seen that at BER $=10^{-3}$, we achieve a 5-dB gain by increasing from $K=2$ to $K=5$. 


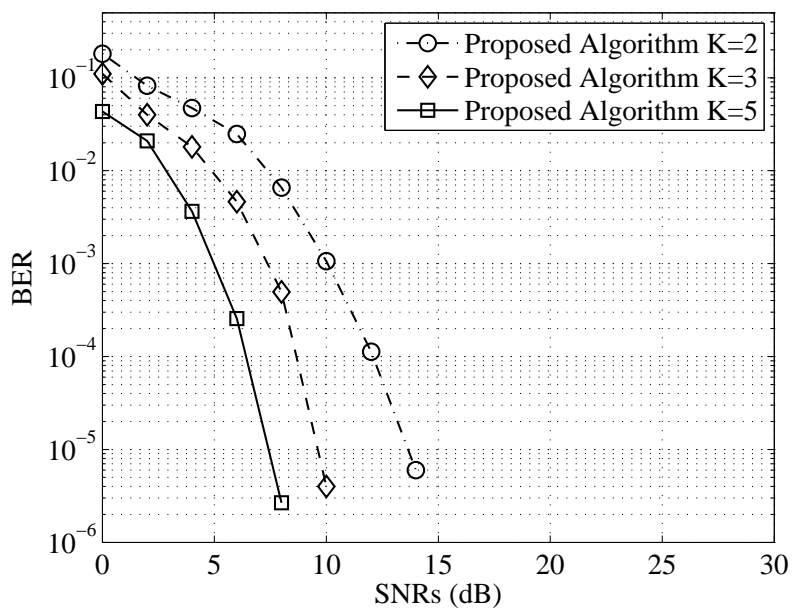

Fig. 4. Example 2. BER versus $\mathrm{SNR}_{\mathrm{S}}$ with varying $K$.

\section{CONCLUSIONS}

In this paper, we have derived the optimal structure of the source precoding matrix and the relay amplifying matrices for parallel MIMO relay communication systems when the nonlinear MMSE-DFE receiver is used at the destination node. The proposed source and relay matrices jointly diagonalize the source-relay-destination channel up to two rotation matrices and minimize the MSE of the signal waveform estimation. The proposed algorithm has an improved BER performance compared with existing techniques.

\section{REFERENCES}

[1] H. Bolukbasi, H. Yanikomeroglu, H. Falconer, and S. Periyalwar, "On the capacity of cellular fixed relay networks," in Proc. Canadian Conf. Electrical and Computer Engr., May 2004, vol. 4, pp. 2217-2220.
[2] R. Pabst, B. H. Walke, D. C. Schultz, D. C. Herhold, H. Yanikomeroglu, S. Mukherjee, H. Viswanathan, M. Lott, W. Zirwas, M. Dohler, H. Aghvami, D. D. Falconer, and G. P. Fettweis, "Relay-based deployment concepts for wireless and mobile broadband radio," IEEE Commun. Mag., vol. 42, pp. 80-89, Sep. 2004.

[3] B. Wang, J. Zhang, and A. Høst-Madsen, "On the capacity of MIMO relay channels," IEEE Trans. Inf. Theory, vol. 51, pp. 29-43, Jan. 2005.

[4] T. Tang, C. B. Chae, R. W. Heath. Jr., "On achievable sum rates of a multiuser MIMO relay channel," in Proc. IEEE ISIT, Seattle, WA, USA, Jul. 2006, pp. 1026-1030.

[5] X. Tang and Y. Hua, "Optimal design of non-regenerative MIMO wireless relays, "IEEE Trans. Wireless Commun., vol. 6, pp. 1398-1407, Apr. 2007.

[6] O. Muñoz-Medina, J. Vidal, and A. Agustín, "Linear transceiver design in nonregenerative relays with channel state information, "IEEE Trans. Signal Process., vol. 55, pp. 2593-2604, Jun. 2007.

[7] W. Guan and H. Luo, "Joint MMSE transceiver design in nonregenerative MIMO relay systems," IEEE Commun. Lett., vol. 12, pp. 517-519, Jul. 2008.

[8] Y. Rong, "Linear non-regenerative multicarrier MIMO relay communications based on MMSE criterion," IEEE Trans. Commun., vol. 58, pp. 1918-1923, Jul. 2010.

[9] Z. Fang, Y. Hua, and J. C. Koshy, "Joint source and relay optimization for a non-regenerative MIMO relay," in Proc. IEEE Workshop Sensor Array Multi-Channel Signal Process., Waltham, WA, Jul. 2006, pp. 239243.

[10] Y. Rong and Y. Hua, "Optimality of diagonalization of multi-hop MIMO relays," IEEE Trans. Wireless Commun., vol. 8, pp. 6068-6077, Dec. 2009.

[11] Y. Rong, X. Tang, and Y. Hua, "A unified framework for optimizing linear non-regenerative multicarrier MIMO relay communication systems," IEEE Trans. Signal Process., vol. 57, pp. 4837-4851, Dec. 2009.

[12] A. S. Behbahani, R. Merched, and A. M. Eltawil, "Optimizations of a MIMO relay network," IEEE Trans. Signal Process., vol. 56, pp. 50625073, Oct. 2008 .

[13] A. Toding, M. R. A. Khandaker, and Y. Rong, "Optimal joint source and relay beamforming for parallel MIMO relay networks," in Proc. 6th Int. Conf. Wireless Commun., Networking and Mobile Computing, Chengdu, China, Sep. 23-25, 2010.

[14] Y. Rong, "Optimal linear non-regenerative multi-hop MIMO relays with MMSE-DFE receiver at the destination," IEEE Trans. Wireless Commun., vol. 9, pp. 2268-2279, Jul. 2010.

[15] A. W. Marshall and I. Olkin, Inequalities: Theory of Majorization and Its Applications. Academic Press, 1979.

[16] Y. Jiang, W. Hager, and J. Li, "The generalized triangular decomposition," Mathematics of Computation, vol. 77, pp. 1037-1056, Apr. 2008. 